\title{
Diseño de un programa de vigilancia epidemiológica para desórdenes Osteomusculares
}

\section{Design of a program of epidemiological surveillance for musculoskeletal disorders}

\author{
Gloria Liliana García Marín \\ Especialista en Gerencia de la Seguridad y Salud en el Trabajo, Corporación Universitaria Iberoamericana. Servicio \\ Nacional de Aprendizaje SENA. Centro de la industria, la empresa y los servicios, Regional Norte Santander \\ Email: glorialgm4210@gmail.com
}

Doi: https://doi.org/10.22267/rceilat.214849.100

\section{Resumen}

Hoy por hoy los desórdenes osteomusculares generados por actividades laborales se presentan con más frecuencia, tienen un alto grado de incapacidad y afectan no solo al trabajador que lo adolece, sino también, a la empresa por el impacto económico que debe asumir. Este documento facilita el proceso para el diseño de un programa de vigilancia epidemiológica para desórdenes osteomusculares, en él, se dan a conocer el proceso de recolección de la información para el diseño del programa y los instrumentos aplicados, se conocen los factores de riesgo y la importancia de que los colaboradores trabajen conjuntamente con la organización en pro de garantizar la salud y bienestar de los integrantes de la organización y la organización en sí misma.

Palabras Claves: Riesgos laborales; Osteomuscular; Ergonomía; Epidemiológica.

\begin{abstract}
Today, musculoskeletal disorders generated by work activities occur more frequently, have a high degree of disability and affect not only the worker who suffers it, but also the company due to the economic impact that it must assume. This document facilitates the process of designing an epidemiological surveillance program for musculoskeletal disorders, in it, the information gathering process for the design of the program and the applied instruments are known, risk factors and the importance of that collaborators work together with the organization in order to guarantee the health and well-being of the members of the organization and the organization itself.
\end{abstract}

Keywords: Musculoskeltal; Occupation risks; Ergonomics; Epidemiology. 


\section{Introducción}

Según estimaciones de la OIT, cada año se producen 2,34 millones de muertes de origen profesional y 2,02 millones de ellas se deben a enfermedades profesionales, lo cual equivale a 5.000 muertes diarias. Todo ello causa grandes sufrimientos a los trabajadores y a sus familias y provoca importantes pérdidas económicas para las economías y las sociedades. Muchas de estas tragedias se pueden evitar poniendo en práctica medidas de prevención, información y formación, medidas adecuadas de inspección, y gracias a un verdadero compromiso con la seguridad y salud en el trabajo por parte de los gobiernos, los empleadores y los trabajadores. Los costos económicos conexos son colosales a nivel de la empresa, a nivel nacional y a nivel mundial. Se estima que las pérdidas que representan los pagos por indemnización, los días de trabajo perdidos, la interrupción de la producción, la formación y la readaptación de profesionales, los gastos médicos, etc., suelen representar aproximadamente un 4\% del PIB. En muchos países la situación ha venido mejorando de manera continua, pero demasiado lenta, y por esta razón los accidentes del trabajo, las enfermedades profesionales y los accidentes mayores siguen siendo demasiado frecuentes y siguen causando grandes sufrimientos humanos y pérdidas económicas. (MEN, 2017).

Los desórdenes osteomusculares son patologías que tienen una alta ocurrencia en los registros estadís- ticos de incapacidades laborales del Sistema General de Riesgos Laborales con un 51,9\% de incidencia; de este valor porcentual el 52\% corresponde al género masculino; (Pino Castillo \& Ponce Bravo , 2017). La empresa "Consolidando Sueños S.A.S" no es ajena a esta situación y los colaboradores suman a dichos porcentajes, por los riesgos a los que están expuestos.

La salud es un bien de interés público y en consecuencia el estado debe generar legislación en pro de la protección de esta. En Colombia se cuenta con una amplia legislación en torno a la seguridad y salud en el trabajo y gran parte está direccionada a la prevención, al diseño y generación de planes que permitan disminuir morbilidades en los equipos de trabajo.

Según Kumar el mecanismo de aparición de las lesiones musculoesqueléticas es de naturaleza biomecánica; cuatro teorías explican el mecanismo de aparición y pueden interrelacionarse o presentarse aisladas en la evolución de un trastorno osteomuscular, dichas teorías son:

- La teoría de interacción multivariante en la cual el trastorno mecánico de un sistema biológico depende de los componentes individuales y sus propiedades mecánicas, las cuales son casualmente afectadas por dotaciones genéticas, características morfológicas, composición psicosocial y riesgos laborales biomecánicos.

- La teoría diferencial que se ex- 
plica por desequilibrio y asimetría en actividades laborales creando fatigas diferenciales, y de ese modo desequilibrio cinético y cinemático en diferentes articulaciones precipitando la aparición de lesiones.

- La teoría de la carga acumulativa sugiere que un rango de carga y un producto de repetición más alto que no permite la recuperación de los tejidos, deja una carga residual que precipita las lesiones.

- La teoría de sobre-esfuerzo que indica que el exceso de esfuerzo precipita al límite de tolerancia en las lesiones laborales locomotoras. (Ordoñez, C. Gómez, E. \& Calvo, A. 2016)

La prevención es la principal herramienta que usan las empresas para lograr impacto positivo en los empleados, disminuyendo patologías de origen laboral y con ello dando cumplimiento a parte de la normatividad que les asiste.

Según datos y cifras publicados por la Organización Mundial de la Salud los trastornos osteomusculares son la principal causa de discapacidad y el dolor lumbar es la causa más frecuente de discapacidad en el mundo; estos trastornos limitan enormemente la movilidad y la destreza, son causa de jubilaciones anticipadas y están relacionadas con depresión. (Organización Mundial de la Salud, 2019)

En Colombia las ARL son el conjunto de entidades públicas y priva- das, normas y procedimientos, destinados a prevenir, proteger y atender a los trabajadores de los efectos de las enfermedades y los accidentes que puedan ocurrirles con ocasión o como consecuencia del trabajo que desarrollan. El Sistema General de Riesgos Profesionales forma parte del Sistema de Seguridad Social Integral establecido por la Ley 100 de 1993. Las disposiciones vigentes de salud ocupacional relacionadas con la prevención de los accidentes de trabajo, enfermedades profesionales y el mejoramiento de las condiciones de trabajo, con las modificaciones previstas en el decreto 1295 de 1994 hacen parte integral del Sistema General de Riesgos Profesionales. (Secretaria Senado, 2020)

Para las ARL el que en las empresas implementen programas de prevención en desórdenes osteomusculares tiene como resultado la disminución de las probabilidades que se presenten morbilidades y comorbilidades en torno a dicha afección y esto no solo es beneficio para la empresa en términos de productividad, disminución de incapacidades, de costos por ausentismo, sino que además promueve el auto cuidado, el bienestar entre los colaboradores, genera ambientes saludables y puede establecerse como una Empresa Saludable. La intervención con este tipo de actividades de prevención, también produce un impacto positivo desde la Responsabilidad Social Empresarial.

El componente de RSE involucra aspectos internos y externos de la empresa, y van directamente relaciona- 
dos con el equipo de trabajo, personal directivo, inversionistas, comunidad, entre otros. Por lo tanto el desarrollo de planes y programas de prevención en el componente de seguridad y salud en el trabajo conlleva un impacto positivo de la empresa en el entorno social, logrando construir un perfil de empresa garante de bienestar para sus colaboradores y por consiguiente para el grupo familiar y el entorno social del trabajador. (Dirección General de Riesgos Profesionales del Ministerio de la Protección Social, 2006) (Díaz, Marziale, Robazzi, \& Freitas, 2010) (Bayer \& Pavas, 2017) (López, A., Ojeda, J., \& Riosm M. 2017)

El objeto del diseño e implementación de un programa de vigilancia epidemiológica para desórdenes osteomusculares asociados a actividades laborales permite a la empresa generar una alerta temprana en la identificación de los orígenes de la enfermedad y con ello tomar acciones de control que permitan atender el riesgo de manera oportuna, en la situación individual, en el desarrollo de la tarea, realizar los ajustes al puesto de trabajo; se debe tener en cuenta que el control del riesgo se debe realizar en el ambiente laboral, monitorear la tendencia y eliminar el riesgo; es importante tener presente que desde la ergonomía se debe ajustar el puesto de trabajo al trabajador. (Federal Institute for Occupational Safety and Health, 2017)

Según la problemática que se presenta en torno a las enfermedades osteomusculares, es importante generar programas con el direccionamiento de la prevención, teniendo como base de partida las matrices de riesgos que segmentan las tendencias epidemiológicas de la población a estudiar en la organización. Para la empresa en la que se adelantó el presente estudio se encontró que al momento de levantar la información, esta no registra enfermedades profesionales en estudio o en calificación, pero los colaboradores si refieren presentar sintomatología asociada a las afecciones osteomusculares, razón por la cual es importante implementar el proceso de vigilancia epidemiológica para desórdenes osteomusculares.

Muchos trastornos osteomusculares pueden prevenirse mediante intervenciones ergonómicas que modifiquen el trabajo y los lugares de trabajo a partir de la evaluación de los factores de riesgo, (FACTS, 2000) esto ya está incorporado en la legislación colombiana, asimismo se precisan las orientaciones para la prevención.

\section{Materiales y Métodos}

Estudio descriptivo analítico, aplicado a 30 colaboradores que laboran en los diferentes restaurantes de la empresa Consolidando Sueños S.A.S. El estudio está orientado a la estructuración (diseño) de un programa de vigilancia epidemiológica para desórdenes osteomusculares. Una vez se obtuvo el permiso por parte de los directivos de la empresa, se continuó con el acercamiento a la población objeto, es decir, quienes trabajan directamente para la empresa, en turnos rotativos. 
Para dar inicio al estudio, el primer proceso implementado fue un diagnóstico, este se llevó a cabo con la aplicación de un método de evaluación de posturas forzadas, REBA (Rapid Entire Body Assessment), este es un método que evalúa el riesgo de posturas estáticas y dinámicas (acciones repetidas, como por ejemplo repeticiones que superan las 4 veces por minuto), se excluye el caminar, acciones adoptadas por los brazos, antebrazos, muñecas, tronco, cuello, piernas. (Ergonómicos, 2015) (Diego-Mas, 2020)

La metodología REBA divide el cuerpo en dos grupos; un grupo A que incluye las piernas, tronco y cuello; un grupo B que comprende miembros superiores como brazos, antebrazos y muñecas. La metodología tiene definidas unas mediciones conexas a una puntuación de cada zona del cuerpo, y en función de dichas puntuaciones se asignan valores: los resultados de estos valores nos llevan a identificar el nivel de actuación frente a la posición evaluada. La metodología se aplicó en una guía para cada grupo de trabajo. (Infopreben, 2000)

El segundo proceso fue la revisión de las condiciones generales de salud del grupo de colaboradores participantes, información que reposa en los archivos de la empresa y que se ha construido durante los procesos de vinculación del personal a la organización y a través de controles y seguimiento médico.

Acto seguido y con el fin de identificar los factores de riesgo ergonó- micos y la sintomatología osteomuscular, se aplicó un instrumento que contiene 11 ítems (para el presente estudio se aplicaron 5 ítems) para la recolección y análisis de información sobre los factores de riesgo ergonómicos y daños a la salud derivados del trabajo, que puedan presentar los trabajadores en diferentes zonas del cuerpo, también las posturas y acciones propias del trabajo.

Los aspectos evaluados fueron la presencia de dolor, la frecuencia de éste, la causa que consideran les genera el dolor o la molestia, también se evaluó el tiempo que estaban expuestos a posiciones de inclinación de cuello y tronco, manos extendidas, muñecas dobladas, presión con los pies.

El instrumento fue desarrollado por la Fundación para la Prevención de riesgos laborales, este cuestionario pretende identificar síntomas y factores de riesgo ergonómicos existentes en los puestos de trabajo seleccionados para el análisis. . (ERGOPAR, 2012)

A los participantes del estudio se les socializó el objetivo del proceso; una vez entendido y aceptado, firmaron el consentimiento informado, se conservó el rigor ético y metodológico (Ministerio de Salud, 1993) La información recogida se transcribió a hojas electrónicas y se usó la estadística descriptiva para la exposición de los datos.

\section{Resultados}

Los 30 trabajadores que participaron del estudio, ocupan los siguientes 
cargos: 13 producción, 13 despachos y 4 administradores; de los cuales el $59 \%$ es femenino y el $41 \%$ es masculino; la edad promedio es de 30 años y llevan trabajando en la empresa el $37 \%$ menos de 1 año, el $23 \%$ entre 1,1 años y 2 años, el 19\% entre 2,1 y 3 años, 16\% entre 3,1 años y 5 años y más de 5,1 años el 5\%.

Con la aplicación de la metodología REBA se evaluaron las condiciones de trabajo en cuanto a carga postural y se estimó el riesgo de padecer desórdenes osteomusculares relacionados con el trabajo y la exposición de los trabajadores. El resultado de este primer análisis indica riesgos bajos y medios; lo que lleva a concluir que la empresa debe iniciar un proceso de implementación de medidas preventivas y correctivas para minimizar el riesgo de que sus colaboradores desarrollen patologías osteomusculares a mediano o largo plazo y evitar un impacto negativo al trabajador, a la empresa y al entorno socio cultural.

Los afecciones registradas son generadas en el desarrollo y cumplimiento de las actividades propias de cada trabajador, estas están inmersas en una serie de acciones repetitivas y de posturas inadecuadas, que conllevan a generar fatiga y con el tiempo ocasionan diagnósticos ocupacionales; uno de los más frecuentes son las afecciones osteomusculares o músculo esqueléticas producidas por movimientos repetitivos y carga postural. (Federal Institute for Occupational Safety and Health, 2017)
En la revisión de las condiciones generales de salud se tiene que un $65 \%$ de los trabajadores participantes del estudio tiene enfermedades no profesionales diagnosticadas; estas son (Ver Tabla 1): Condiciones Generales de Salud.

\begin{tabular}{|c|c|}
\hline \multicolumn{2}{|c|}{ CONDICIONES DE SALUD } \\
\hline $\begin{array}{c}\text { ENFERMEDADES } \\
\text { DIAGNOSTICADAS }\end{array}$ & PORCENTAJE \\
\hline ACALASIA & $2 \%$ \\
\hline AMIGDALITIS & $2 \%$ \\
\hline ASMA & $4 \%$ \\
\hline COLESTEROL & $2 \%$ \\
\hline GASTRITIS & $30 \%$ \\
\hline HIPERTENSION & $2 \%$ \\
\hline HIPOGLICEMIA & $2 \%$ \\
\hline HIPOTIROIDISMO & $6 \%$ \\
\hline MIGRAÑA & $10 \%$ \\
\hline MIOPIA & $1 \%$ \\
\hline RINITIS & $4 \%$ \\
\hline \multicolumn{2}{|c|}{}
\end{tabular}

Tabla 1. Condiciones Generales de salud. Fuente: Archivo de la empresa.

Con relación a la presencia de dolores osteomusculares los resultados obtenidos a través del cuestionario síntomas y factores de riesgo ergonómicos existentes en los puestos de trabajo, en la tabla 2 se encuentra el registro de las molestias o dolores de cuello, hombros, espalda, manos y muñecas, codos, piernas, rodillas y pies, los registros de los trabajadores participantes del estudio refiere que los dolores o molestias registradas en las distintas zonas corporales, se han producido como consecuencia de las tareas propias del puesto de trabajo, así, un 34\% reporta dolor en el cuello, hombro y espalda dorsal, un $46 \%$ en espalda lumbar, $8 \%$ en codos, un $76 \%$ de manos y muñecas, resultado 
conexo a que las manos son la parte corporal más usada en el desarrollo de la funciones, le sigue con un alto porcentaje los pies con un $69 \%$ y esto como efecto de que las jornadas laborales se ejecutan en posición de pie; un 38\% refiere molestias o dolores en piernas y un $8 \%$ en rodillas.

La tabla 3 registra que el 100\% de los trabajadores no desarrollan actividades laborales en posición de sentado o con apoyo lumbar y que las principales actividades requieren de posiciones de pie sin caminar y otras caminando.

La tabla 4 contiene el registro de los tiempos que deben trabajar adoptando posturas específicas de cuello y cabeza, esta componente tiene como resultado que un $92 \%$ de los trabajadores aplican posturas en las cuales deben inclinar el cuello y cabeza hacia adelante, un 48\% debe girar el cuello y cabeza y un 31\% inclina la espalda y tronco hacia adelante.

Los resultados obtenidos de las posturas y acciones propias del trabajo realizado con manos y muñecas, registrado en la tabla 5 tenemos que el $54 \%$ de los trabajadores realizan posturas de manos por encima de la cabeza o codos por encima de los hombros, esta es una posición repetitiva, en este mismo componente un $17 \%$ refiere realizar movimientos de una o ambas muñecas dobladas hacia arriba o hacia abajo y hacia los lados o giradas, se resalta que no se registran actividades que requieren ejercer presión con uno de los pies.
La tabla 6 contiene los resultados de la actividad laboral de manipulación de elementos, este registro muestra que el 33\% de los trabajadores desarrolla actividades donde presionan o levantan objetos o herramientas con los dedos en forma de pinza y otro $33 \%$ agarra o sujeta con fuerza objetos o herramientas con las manos.

Con la información recolectada se realizó la formulación y diseñó del Programa de Vigilancia Epidemiológica para Desórdenes Osteomusculares, el cual se enmarcó en las directrices contenidas en el Decreto No. 1443 que tiene por objeto definir las directrices de obligatorio cumplimiento para implementar el Sistema de Gestión de la Seguridad y Salud en el Trabajo - SG-SST, que deben ser aplicadas por todos los empleadores públicos y privados, los contratantes de personal bajo modalidad de contrato civil, comercial o administrativo, las organizaciones de economía solidaria y del sector cooperativo, las empresas de servicios temporales y tener cobertura sobre los trabajadores dependientes, contratistas, trabajadores cooperados y los trabajadores en misión; en él también se registra que el procedimiento lógico y por etapas que permite el mejoramiento continuo a través de los pasos de PHVA. En el primer paso, se debe PLANIFICAR la forma de mejorar la seguridad y salud de los trabajadores, encontrando qué cosas se están haciendo incorrectamente o se pueden mejorar y determinando ideas para solucionar esos problemas; en el proceso del HACER se realiza la implementación de las medidas plani- 
ficadas; en el VERIFICAR se revisan que los procedimientos y acciones implementados estén logrando los resultados deseados $\mathrm{y}$ en el ACTUAR se realizan las acciones de mejora para obtener los mayores beneficios en la seguridad y salud de los trabajadores. (Ministerio de Trabajo, 2014, pág. 3)

El Programa de Vigilancia Epidemiológica para desórdenes Osteomusculares debe contener:

$\Rightarrow$ Introducción

$\Rightarrow$ Justificación

$\Rightarrow$ Diagnóstico

$\Rightarrow$ Riesgo expresado

$\Rightarrow$ Universo de vigilancia

$\Rightarrow$ Riesgo inherente

$\Rightarrow$ Objetivo del plan

$\Rightarrow$ Objetivos específicos

$\Rightarrow$ Definiciones de los conceptos que se encuentran en el programa

$\Rightarrow$ Metodología a implementar según las fases del PHVA

$\Rightarrow$ Responsabilidades del empleador

$\Rightarrow$ Responsabilidades del equipo de trabajo

$\Rightarrow$ Recomendaciones y conclusiones.

La empresa Consolidando Sueños S.A.S. requiere de la implementación del programa de Vigilancia Epidemiológica para desórdenes osteomusculares, esto, no solo por los resultados obtenidos a través de la investigación, sino además en cumplimiento de la ley, y como garantía del bienestar para el equipo de colaboradores, para lograrlo debe:

$\Rightarrow$ Realizar nuevamente el proceso de inspección frente a las condiciones de salud de los trabajadores, tales como: exámenes médicos laborales, encuestas de morbilidad sentida, entre otros, que puedan aportar mayor información a las investigaciones dirigidas a los sistemas de vigilancia epidemiológica y que permitan obtener resultados precisos de las enfermedades osteomusculares por movimientos repetitivos diagnosticadas o en riesgo de adquirir y por ende intervenir.

$\Rightarrow$ Realizar actividades de promoción y prevención para el sistema de vigilancia epidemiológica para desórdenes osteomusculares dirigido a todos los trabajadores, esto con el objeto de que sean actores activos en cada una de las acciones que se implementan y trabajar en el logro de un mayor impacto y efectividad al proceso.

$\Rightarrow$ Diseñar con el apoyo de profesional en fisioterapia o con apoyo de la ARL (Administradora de Riesgos Laborales) revisiones de puestos de trabajo direccionados a la atención de los desórdenes osteomusculares que refieren los colaboradores, pues estos desórdenes aun no reportan enfermedades en calificación ni un alto impacto de frecuencia de la morbilidad sentida y se está a tiempo de atención.

$\Rightarrow$ En la fase del hacer se recomienda que la empresa implemente actividades de calistenia o pausas activas que conduzcan a mejorar 
la morbilidad sentida en afecciones osteomusculares.

$\Rightarrow$ Se proponen una serie de actividades terapéuticas, la implementación o realización de estas actividades con una frecuencia de cada dos horas en el día las convierten en pausas activas, las cuales son un breve descanso que se lleva a cabo durante la jornada laboral y están direccionadas a mejorar el rendimiento, la eficiencia, la ejecución de la tarea, prevenir enfermedades, pero ante todo permite el autocuidado, el mejoramiento de la salud física y mental, conllevando a disminuir las posibilidades de generar accidentes y/o enfermedades no solo de tipo osteomuscular.

\section{Conclusiones}

$\Rightarrow$ Todos los participantes del estudio realizan trabajos repetitivos, con posturas forzadas y antigravitacionales en los miembros superiores, exponiéndose a desarrollar síntomas o afecciones osteomusculares y la espalda es la zona corporal más afectada.

$\Rightarrow$ Se encuentra una asociación significativa entre el dolor de la espalda lumbar con manos y muñecas; se concluye que es el riesgo de carga física y movimientos repetitivos a los que están sometidos.

$\Rightarrow$ Se observa igualmente una relación muy estrecha entre las afec- ciones de piernas y pies, la cual puede tener conexión por las largas jornadas que realizan trabajando de pie.

$\Rightarrow$ Un porcentaje considerable de participantes del estudio (promedio el 42,7\%) consideran que las morbilidades se han generado en el desarrollo de las tareas del puesto de trabajo que desempeñan.

$\Rightarrow$ En la evaluación de las posturas $\mathrm{y}$ acciones propias del trabajo se registra un alto porcentaje realizando posturas repetitivas.

$\Rightarrow$ Teniendo en cuenta que las afecciones osteomusculares son patologías que afectan tanto extremidades inferiores como superiores y está demostrado que estas afecciones conservan un alto grado de relación con las funciones o tareas que se desempeñan en los distintos puestos de trabajo, ya sea por movimientos repetitivos, manipulación manual de cargas, posturas no adecuadas, movimientos forzados, entre otras posiciones corporales que se toman en la ejecución de la actividad laboral, la empresa debe iniciar la consolidación del Programa de Vigilancia Epidemiológica para desórdenes osteomusculares de manera pronta y hacer frente a las casos que se están presentando. Esto permite minimizar la generación de morbilidades sentidas y afecciones relevantes para los colaboradores que pueden generar 
afecciones importantes a la salud.

$\Rightarrow$ En los desórdenes osteomusculares también es preciso analizar y tener en cuenta condiciones o características individuales de cada trabajador. Algunas de ellas son las preexistencias, la edad, estilos de vida, estos son factores de alta incidencia en la aparición de enfermedades y a los cuales les deben hacer un seguimiento y control.

$\Rightarrow$ De acuerdo con la metodología establecida para el diseño del programa de vigilancia epidemiológica para desórdenes osteomusculares en la empresa Consolidando Sueños S.A.S., se espera que la morbilidad asociada a desórdenes osteomusculares disminuya con las medidas de prevención definidas y que la empresa puede implementar.

\section{Discusión}

En la relación del tiempo que llevan los colaboradores vinculados se encuentra que solo un $5 \%$ tiene más de 5 años en la empresa, los demás tienen entre un mes y 4,11 años, razón por la cual aún no se ven tan ampliamente afectados por problemas osteomusculares en la realización de las tareas propias del puesto y es por esto que es importante la implementación del programa de vigilancia epidemiológica para desórdenes osteomusculares.

Como también se registra en la ta- bla 2 el 38\% del grupo estudiado se ha visto impedido para realizar el trabajo asignado y esta imposibilidad se presentó por afecciones en la zona corporal de muñecas, manos y pies, situación que debe ser revisada y atendida buscando garantizar al trabajador las mejores condiciones de trabajo para su bienestar, teniendo en cuenta que este se extiende a su grupo social.

En la publicación de la Organización Mundial de la Salud refieren que los trastornos osteomusculares afectan a personas de todas las edades y en todas las regiones del mundo. El estudio sobre la carga mundial de morbilidad muestra los efectos de estas enfermedades y la gran discapacidad que producen. Esto demuestra que la promoción y la prevención de estas lesiones tienen como objetivo la reducción de casos, en pro de no sumar y acrecentar la estadística que presenta la patología.

No se puede concebir la seguridad como el simple hecho de cumplir con la legislación, las normas, o el generar planes para evitar accidentes: las empresas deben abordar la seguridad desde una óptica más amplia como la de promover en sus colaboradores la participación activa en la solución de problemas y hasta en el establecer propuestas que permitan mejorar las condiciones de salud y la disminución de riesgos laborales. Orientar en el concepto de que la seguridad y la salud son un valor, es la premisa; la prioridad de garantizar la integridad física, psicológica y el bienestar individual y colectivo. 
La participación activa del empleador y del trabajador genera una conexión y entendimiento de que los nuevos contextos laborales requieren de una constante revisión del riesgo, trabajar en ello con anticipación, tener un análisis profundo de las consecuencias, es decir ir más allá de lo que exige el cumplimiento de la ley o la norma.

Una de las necesidades del ser humano es la seguridad y dentro de esta se contempla la seguridad física, de empleo, de recursos, de salud; esto representa bienestar y trabajar por la conservación de estas necesidades genera en el ser humano compromiso en el ambiente laboral, personal y familiar, conllevando a generar propuestas que benefician a la persona, al grupo de trabajo y por consiguiente a la empresa que cuenta con personal comprometido con el autocuidado, promoviendo soluciones de beneficio conjunto.

\begin{tabular}{|c|c|c|c|c|c|c|c|c|}
\hline \multirow[t]{2}{*}{ Zona del cuerpo } & \multicolumn{2}{|c|}{$\begin{array}{r}\text { Tienes molestia o } \\
\text { dolor en esta zona }\end{array}$} & \multicolumn{2}{|c|}{ Con que frecuencia? } & \multicolumn{2}{|c|}{$\begin{array}{c}\text { Te ha impedido alguna } \\
\text { vez realizar tu trabajo } \\
\text { actual }\end{array}$} & \multicolumn{2}{|c|}{$\begin{array}{l}\text { Se ha producido como } \\
\text { consecuencia de las tareas } \\
\text { del puesto señalado }\end{array}$} \\
\hline & Molestia & Dolor & A Veces & $\begin{array}{l}\text { Muchas } \\
\text { Veces }\end{array}$ & Si & No & Si & No \\
\hline $\begin{array}{l}\text { Cuello - Hombros } \\
\text { y/o espalda dorsal }\end{array}$ & $15 \%$ & $46 \%$ & $23 \%$ & $31 \%$ & $8 \%$ & & $54 \%$ & \\
\hline Espalda Lumbar & $38 \%$ & $8 \%$ & $38 \%$ & $8 \%$ & $8 \%$ & & $46 \%$ & \\
\hline Codos & $8 \%$ & & $8 \%$ & & & & $8 \%$ & \\
\hline Manos y Muñecas & $38 \%$ & $38 \%$ & $38 \%$ & $38 \%$ & $38 \%$ & & $76 \%$ & \\
\hline Piernas & $23 \%$ & $15 \%$ & $23 \%$ & $15 \%$ & $15 \%$ & & $38 \%$ & \\
\hline Rodillas & $15 \%$ & $8 \%$ & $23 \%$ & & & & $8 \%$ & \\
\hline Pies & $38 \%$ & $31 \%$ & $38 \%$ & $31 \%$ & $38 \%$ & & $69 \%$ & \\
\hline
\end{tabular}

Tabla 2. Daños a la Salud derivados del trabajo Fuente: Método de recolección de Información.

Para cada zona corporal indica si tienes molestia o dolor, su frecuencia, si te ha impedido realizar tu trabajo actual y si esa molestia o dolor se ha producido como consecuencia de las tareas que realizas en tu puesto de trabajo.

\begin{tabular}{|l|c|c|c|c|}
\hline & $\begin{array}{c}\text { Nunca (menos } \\
\text { de } 30 \text { min }\end{array}$ & $\begin{array}{c}\text { Entre } \mathbf{3 0} \text { min } \\
\text { y } \mathbf{2} \text { horas }\end{array}$ & $\begin{array}{c}\text { Entre dos y } \\
\text { cuarto horas }\end{array}$ & Más de 4 horas \\
\hline $\begin{array}{l}\text { 1. Sentado silla - Apoyo } \\
\text { Lumbar }\end{array}$ & $100 \%$ & & & \\
\hline 2. De pie sin caminar & $8 \%$ & $8 \%$ & $46 \%$ & $31 \%$ \\
\hline 3. Caminando & $23 \%$ & $31 \%$ & $15 \%$ & $31 \%$ \\
\hline $\begin{array}{l}\text { 4. Caminando subiendo y } \\
\text { bajando niveles (peldaños - } \\
\text { escaleras - rampas, etc.) }\end{array}$ & $62 \%$ & $23 \%$ & $8 \%$ & \\
\hline
\end{tabular}

Tabla 3. Posturas y acciones propias del trabajo

Fuente: Método de recolección de Información 
¿Durante cuánto tiempo tienes que trabajar adoptando o realizando estas posturas?

\begin{tabular}{|c|c|c|c|c|c|c|}
\hline \multirow[t]{2}{*}{ Zona del cuerpo } & \multirow{2}{*}{$\begin{array}{c}\text { Nunca } \\
\text { menos de } \\
30 \\
\text { Minutos }\end{array}$} & \multirow{2}{*}{$\begin{array}{l}\text { Entre } 30 \\
\text { minutos y } \\
\text { dos horas }\end{array}$} & \multirow[t]{2}{*}{$\begin{array}{l}\text { Entre } 2 \text { y } \\
4 \text { horas }\end{array}$} & \multirow[t]{2}{*}{$\begin{array}{c}\text { Mas de } 4 \\
\text { horas }\end{array}$} & \multicolumn{2}{|c|}{$\begin{array}{c}\text { Esta postura se repite con } \\
\text { frecuencia es decir cada pocos } \\
\text { segundos ó es fija todo el tiempo }\end{array}$} \\
\hline & & & & & La repito & Es fija \\
\hline $\begin{array}{c}\text { Inclinar el } \\
\text { cuello/cabeza hacia } \\
\text { adelante }\end{array}$ & $8 \%$ & $15 \%$ & $8 \%$ & $69 \%$ & $92 \%$ & \\
\hline $\begin{array}{c}\text { Inclinar el } \\
\text { cuello/cabeza hacia } \\
\text { atrás }\end{array}$ & & & & & & \\
\hline $\begin{array}{l}\text { Inclinar el cuello } \\
\text { cabeza hacia uno o } \\
\text { ambos lados }\end{array}$ & & & & & & \\
\hline $\begin{array}{c}\text { Girar el } \\
\text { cuello/cabeza }\end{array}$ & $54 \%$ & $8 \%$ & $31 \%$ & $8 \%$ & $46 \%$ & \\
\hline $\begin{array}{l}\text { Inclinar la espalda } \\
\text { Tronco hacia } \\
\text { adelante }\end{array}$ & $62 \%$ & $23 \%$ & $8 \%$ & $8 \%$ & $31 \%$ & \\
\hline $\begin{array}{l}\text { Inclinar la espalda } \\
\text { Tronco hacia atrás }\end{array}$ & & & & & & \\
\hline $\begin{array}{l}\text { Inclinar la espalda } \\
\text { /tronco hacia un } \\
\text { lado o hacia ambos }\end{array}$ & & & & & & \\
\hline $\begin{array}{c}\text { Girar la espalda } \\
\text { tronco }\end{array}$ & $77 \%$ & $8 \%$ & $8 \%$ & $8 \%$ & $23 \%$ & \\
\hline
\end{tabular}

Tabla 4. Posturas y acciones propias del trabajo

Fuente: Método de recolección de Información

¿Durante cuánto tiempo tienes que trabajar adoptando o realizando estas posturas de cuello/cabeza?

\begin{tabular}{|c|c|c|c|c|c|c|}
\hline \multirow[t]{2}{*}{ Zona del cuerpo } & \multirow{2}{*}{$\begin{array}{c}\text { Nunca } \\
\text { menos de } \\
30 \text { Minutos }\end{array}$} & \multirow{2}{*}{$\begin{array}{l}\text { Entre } 30 \\
\text { minutos y } \\
\text { dos horas }\end{array}$} & \multirow{2}{*}{$\begin{array}{l}\text { Entre } 2 \text { y } 4 \\
\text { horas }\end{array}$} & \multirow{2}{*}{$\begin{array}{c}\text { Mas de } 4 \\
\text { horas }\end{array}$} & \multicolumn{2}{|c|}{$\begin{array}{l}\text { Esta postura se repite con } \\
\text { frecuencia es decir cada pocos } \\
\text { segundos ó es fija todo el }\end{array}$} \\
\hline & & & & & La repito & Es fija \\
\hline $\begin{array}{l}\text { Las manos por encima de } \\
\text { la cabeza o los codos por } \\
\text { encima de los hombros }\end{array}$ & $33 \%$ & $17 \%$ & & $17 \%$ & $54 \%$ & \\
\hline $\begin{array}{c}\text { Una o ambas muñecas } \\
\text { dobladas hacia arriba } \\
\text { o hacia abajo, hacia los } \\
\text { lados o giradas (giro } \\
\text { de antebrazo) } \\
\end{array}$ & $33 \%$ & & $17 \%$ & $17 \%$ & $17 \%$ & \\
\hline $\begin{array}{l}\text { Ejerciendo presión } \\
\text { con uno de los pies }\end{array}$ & & & & & & \\
\hline
\end{tabular}

Tabla 5. Posturas y acciones propias del trabajo

Fuente: Método de recolección de Información 
¿Durante cuánto tiempo tienes que trabajar adoptando o realizando estas posturas de manos por encima de la cabeza o codos por encima de hombros?

\begin{tabular}{|c|c|c|c|c|c|c|}
\hline \multirow[t]{2}{*}{ Zona del cuerpo } & \multirow{2}{*}{$\begin{array}{c}\text { Nunca } \\
\text { menos de } \\
30 \text { Minutos }\end{array}$} & \multirow{2}{*}{$\begin{array}{l}\text { Entre } 30 \\
\text { minutos y } \\
\text { dos horas }\end{array}$} & \multirow{2}{*}{$\begin{array}{c}\text { Entre } 2 \text { y } 4 \\
\text { horas }\end{array}$} & \multirow{2}{*}{$\begin{array}{c}\text { Mas de } 4 \\
\text { horas }\end{array}$} & \multicolumn{2}{|c|}{$\begin{array}{l}\text { Esta postura se repite con } \\
\text { frecuencia es decir cada } \\
\text { pocos segundos ó es fija }\end{array}$} \\
\hline & & & & & La repito & Es fija \\
\hline $\begin{array}{c}\text { Sosteneer presionar o } \\
\text { levantar objetos o } \\
\text { herramientas con los } \\
\text { dedos en forma de pinza }\end{array}$ & $50 \%$ & & & $33 \%$ & $33 \%$ & \\
\hline $\begin{array}{c}\text { Utilizar de manera } \\
\text { intensiva los dedos } \\
\text { (ordenador, controles, } \\
\text { botoneras, controles } \\
\text { remotos, cajas } \\
\text { registradoras) }\end{array}$ & & & & & & \\
\hline $\begin{array}{l}\text { Agarrar o sujetar con } \\
\text { fuerza objetos o } \\
\text { herramientas con las } \\
\text { manos }\end{array}$ & $50 \%$ & & $17 \%$ & $33 \%$ & $33 \%$ & \\
\hline $\begin{array}{c}\text { Utilizar de manera } \\
\text { intensiva los dedos } \\
\text { (ordenador, controles, } \\
\text { botoneras, controles } \\
\text { remotos, cajas } \\
\text { registradoras) }\end{array}$ & & & & & & \\
\hline $\begin{array}{c}\text { Agarrar o sujetar con } \\
\text { fuerza objetos o } \\
\text { herramientas con las } \\
\text { manos }\end{array}$ & & & & & & \\
\hline
\end{tabular}

Tabla 6. Posturas y acciones propias del trabajo Fuente: Método de recolección de Información

¿Durante cuánto tiempo tienes que trabajar adoptando o realizando posturas de presionar o levantar objetos o herramientas con los dedos en forma de pinza?

\section{Bibliografía}

Bayer, C. M., \& Pavas, F. A. (2017). docplayer. Obtenido de Sistema de Vigilancia Epidemiológica para Rieskgo Osteomuscular Asociado a Actividades Laborales en Empresas del Sector Metalmecánico.

Díaz, M. d., Marziale, M. H., Robazzi, M. L., \& Freitas, F. C. (2010). Lesiones osteomusculares en trabajadores de un hospital mexicano y la ocurrencia del ausentismo. Ciencia y Enfermería. 
Diego-Mas, J. A. (28 de Octubre de 2020). Universitar Politécnica de Valencia . Obtenido de Ergonautas : http://www.ergonautas.upv.es/metodos/reba/reba-ayuda.php

Dirección General de Riesgos Profesionales del Ministerio de la Protección Social. (2006). Guía de Atención Integral Basada en la Evidencia para Desórdenes Músculo Esqueléticos Relacionados con Movimientos Repetitivos de Miembros Superiores. Bogotá.

Ergonómicos, E. E. (30 de Diciembre de 2015). Método reba evita las lesiones postulares Obtenido de http://www.ergoibv.com/blog/metodo-reba-evita-las-lesiones-posturales-2/

ERGOPAR. (2012). Un procedimiento de Ergonomía Participativa para la Prevención de Trastornos Musculoesqueléticos de Origen Laboral. Madrid: Istas.

FACTS, A. E. (10 de 2000). European Agency for Safety and health at Work. Obtenido de https://osha.europa.eu/es/publications/factsheets/3

Federal Institute for Occupational Safety and Health. (2017). Prevención de los Trastornos Musculo Esqueléticos en el Lugar de Trabajo. Alemania: Ifado.

Infopreben. (30 de 06 de 2000). Infopreben Prevención riesgos laborales en el sectro primario. Obtenido de http://www.infopreben.com/index.php/riesgos-itsaspreben/ item/364-excel-para-aplicaci\%C3\%B3n-del-m\%C3\%A9todo-reba-de-evaluaci\%C3\%B3n-ergon\%C3\%B3mica

MEN, T. R. (29 de 09 de 2017). International Labour Organization Obtenido de https:// www.ilo.org/wcmsp5/groups/public/---ed_norm/---normes/documents/genericdocument/wcms_569995.pdf

Ministerio de Salud. (04 de 10 de 1993). Resolución numero 8430 de 1993. Obtenido de https:/www.minsalud.gov.co/sites/rid/Lists/BibliotecaDigital/RIDE/DE/DIJ/RESOLUCION-8430-DE-1993.PDF

Ministerio de Trabajo. (2014). Decreto 1443 de 2014. En Objeto Campo de Aplicación y Definiciones (págs. 5 - 6). Bogotà: Ministerio del Trabajo.

Ordoñez, C., Goméz, E. \& Calvo, A. (2016). Desórdenes músculo esqueléticos relacionados con el trabajo. Revista Colombiana de Salud Ocupacional, 6(1) 27-32.

Organización Mundial de la Salud. (09 de Agosto de 2019). World Health Organization. Obtenido de https://www.who.int:https://www.who.int/es/news-room/fact-sheets/ detail/musculoskeletal-conditions

Pino Castillo, S., \& Ponce Bravo, G. (2017). Comportamiento de la Emfermedad Laboral en Colombia 2015 2017. Bogota: Revista Fasecolda.

Lopez, A., Ojeda, J., \& Riosm M. (2017). La responsabilidad social empresarial desde la percepción del capital humano. Estudio de un caso. Revista de Contabilidad, 20(1) 36-46.

Secretaría Senado . (15 de Octubre de 2020). Decreto 1295 de 1994. República de Colombia 